\title{
О природе аномальной температурной зависимости коэффициента Холла, наблюдающейся в полупроводниковых кристаллах твердых растворов $\mathrm{Bi}_{2} \mathrm{Te}_{3}-\mathrm{Sb}_{2} \mathrm{Te}_{3}$
}

\author{
(C) Н.П. Степанов \\ Забайкальский государственный университет, \\ 672039 Чита, Россия \\ E-mail: NP-Stepanov@mail.ru
}

Поступила в Редакцию 12 апреля 2018 г.

В окончательной редакции 5 сентября 2019 г.

Принята к публикации 9 сентября 2019 г.

На основе анализа результатов экспериментальных исследований показано, что в ряде кристаллов $\mathrm{Bi}_{2} \mathrm{Te}_{3}-\mathrm{Sb}_{2} \mathrm{Te}_{3}$, в которых величина электропроводности при $4.2 \mathrm{~K}$ превышает $1.5 \cdot 10^{6} \mathrm{CM} / \mathrm{M}$, интенсифицируется определенный процесс, обеспечивающий рост электропроводности в диапазоне от 4.2 до $15 \mathrm{~K}$. Последующее уменьшение величины электропроводности с ростом температуры от $15 \mathrm{~K}$ и до начала доминирования собственной проводимости, сопровождающееся аномальным увеличением коэффициента Холла, может быть частично обусловлено снижением интенсивности указанного процесса. Сформулировано предположение о том, что в условиях сближения энергии плазмона и ширины запрещенной зоны влияние плазмон-фонон-поляритонов способно обеспечить обратное воздействие поляризации, составляющее основу этого процесса.

Ключевые слова: полупроводники, электропроводность, коэффициент Холла, плазма свободных носителей заряда, плазмон-фонон-поляритоны, ширина запрещенной зоны.

DOI: $10.21883 /$ FTP.2020.01.48761.8890

\section{1. Введение}

В ряде полупроводниковых материалов, отличающихся сравнительно высокой электропроводностью и концентрацией свободных носителей заряда, таких, например, как $\mathrm{PbTe}, \mathrm{Sb}_{2} \mathrm{Te}_{3}$, наблюдается сильный, в 2 -4 раза, рост коэффициента Холла $R_{\mathrm{H}}$ при изменении температуры от $50-80 \mathrm{~K}$ до начала перехода к собственной проводимости. В кристаллах твердых растворов $\mathrm{Bi}_{2} \mathrm{Te}_{3}-\mathrm{Sb}_{2} \mathrm{Te}_{3}$ такое аномальное увеличение коэффициента Холла наблюдается при любом соотношении компонент $\mathrm{Bi}_{2} \mathrm{Te}_{3}$. Однако если в $p$-типе $\mathrm{Bi}_{2} \mathrm{Te}_{3}$ происходит увеличение $R_{\mathrm{H}}$ в 1.5 раза, то в $p$-типе $\mathrm{Sb}_{2} \mathrm{Te}_{3}$ уже в 4 раза. Причем в $\mathrm{Bi}_{2} \mathrm{Te}_{3}$ оно наблюдается как в $p$-, так и $n$-типе и оказывается чувствительно к количеству и типу внесенной в кристалл примеси, исчезая в кристаллах с относительно малой концентрацией свободных носителей заряда. В широко известной работе [1] отмечено, что не существует убедительного объяснения аномального температурного поведения коэффициента Холла в $n$-типе $\mathrm{Bi}_{2} \mathrm{Te}_{3}$. Несмотря на появившиеся в более позднее время сведения о сложном строении зоны проводимости этого материала, которые можно было бы использовать для объяснения этого явления, по аналогии с тем как это принято для $p$-типа $\mathrm{Bi}_{2} \mathrm{Te}_{3}$, детальных расчетов, однозначно объясняющих природу аномальной температурной зависимости коэффициента Холла в этих материалах, не производилось. В данной работе приведены результаты исследования спектров коэффициента отражения $R$ от монокристаллов $\mathrm{Bi}_{2} \mathrm{Te}_{3}-\mathrm{Sb}_{2} \mathrm{Te}_{3}$, полу- ченных при различных температурах в спектральной области проявления эффектов, обусловленных плазмой свободных носителей заряда, которые в совокупности с экспериментальными данными о температурном поведении электропроводности, полученными рядом исследователей, позволяют вернуться к обсуждению природы данного явления.

\section{2. Методика и техника эксперимента}

Исследовались монокристаллы твердых растворов системы $\mathrm{Bi}_{2} \mathrm{Te}_{3}-\mathrm{Sb}_{2} \mathrm{Te}_{3}$, содержащие 0, 10, 25, 40, 50, 60, $70,80,90$ и 100 мол\% $\mathrm{Sb}_{2} \mathrm{Te}_{3}$, выращенные методом Чохральского в Институте металлургии и материаловедения им. А.А. Байкова РАН. В качестве исходных материалов использовались Те, $\mathrm{Sb}, \mathrm{Bi}$, содержащие 99.9999 мас \% основного вещества. В оптическом отношении твердые растворы $\mathrm{Bi}_{2} \mathrm{Te}_{3}-\mathrm{Sb}_{2} \mathrm{Te}_{3}$ являются одноосными кристаллами. Диэлектрическая проницаемость в них определяется тензором второго ранга с двумя независимыми компонентами $\varepsilon_{\perp}$ и $\varepsilon_{\|}$, соответствующими перпендикулярной и параллельной ориентации вектора напряженности электрического поля $E$ относительно оптической оси кристалла $C_{3}$. В связи с этим регистрировались спектры отражения неполяризованного и поляризованного излучения как от свежеприготовленного скола кристалла по плоскости спайности, обладающей зеркальным блеском, так и от полированной поверхности, содержащей оптическую ось кристалла $C_{3}$. 
Угол падения излучения на образец не превышал $8^{\circ}$. При регистрации спектра от скола кристалла вектор $E$ падающего на кристалл электромагнитного излучения практически перпендикулярен оптической оси кристалла $C_{3}\left(\mathbf{k} \| C_{3}, \mathbf{E} \perp C_{3}\right)$, где $\mathbf{k}-$ волновой вектор падающего излучения. В этом случае возможно определение одной из компонент тензора диэлектрической проницаемости $\varepsilon_{\perp}$. Для определения другой компоненты $\varepsilon_{\|}$ необходимо измерение спектра отражения от плоскости, содержащей ось $C_{3}$, с применением поляризованного излучения в геометрии $\mathbf{k} \perp C_{3}, \mathbf{E} \| C_{3}$. Далее везде индекс $\perp$ означает, что $\mathbf{E} \perp C_{3}$.

Регистрация спектров коэффициента отражения $R(v)$ в диапазоне температур от 80 до $300 \mathrm{~K}$ была выполнена на фурье-спектрометре BRUKER IFS-113V с разрешением $1 \mathrm{~cm}^{-1}$

\section{3. Обсуждение результатов}

Спектры отражения инфракрасного излучения от кристалла $\mathrm{Bi}_{1.5} \mathrm{Sb}_{0.5} \mathrm{Te}_{3}$, полученные при температурах 80, 150,200 и $300 \mathrm{~K}$, представлены на рис. 1, из которого видно, что минимум коэффициента отражения, обусловленный плазменным резонансом свободных носителей заряда, с ростом температуры смещается в длинноволновую часть спектра. Температурные изменения спектров отражения других исследованных кристаллов имеют аналогичный характер. В ходе анализа спектров отражения при помощи соотношений Крамерса-Кронига были определены плазменные частоты $\omega_{p}$ и значения одной из компонент тензора высокочастотной диэлектрической проницаемости $\varepsilon_{\perp}$, значения которых для монокристалла $\mathrm{Bi}_{1.5} \mathrm{Sb}_{0.5} \mathrm{Te}_{3}$ приведены в таблице. Используя экспериментально определенные $\omega_{p}$ и $\varepsilon_{\perp}$, можно в соответствии с выражением

$$
\omega_{p}^{2}=\frac{p e^{2}}{m^{*} \varepsilon_{0} \varepsilon_{\perp}}
$$

в котором $e-$ заряд электрона, $p-$ концентрация, $m^{*}$ - эффективная масса дырок, $\varepsilon_{0}$ - диэлектрическая постоянная, рассчитать отношение $p / m^{*}$, также приведенное в таблице, из которой видно, что с ростом температуры от 80 до $300 \mathrm{~K}$ наблюдается уменьшение отношения $p / m^{*}$ в 1.5 раза.

Экспериментальные значения $\omega_{p \perp}, \varepsilon_{\infty}, \sigma$ и результаты расчета $p / m^{*}$ для кристалла $\mathrm{Bi}_{1.5} \mathrm{Sb}_{0.5} \mathrm{Te}_{3}$. Значения $\sigma$ соответствуют данным, представленным в работе [4]

\begin{tabular}{r|c|c|c|c}
\hline$T, \mathrm{~K}$ & $\omega_{p \perp} \cdot 10^{-13}, \mathrm{c}^{-1}$ & $\varepsilon_{\infty \perp}$ & $\begin{array}{c}\sigma \cdot 10^{-6}, \\
\mathrm{OM}^{-1} \cdot \mathrm{M}^{-1}\end{array}$ & $\begin{array}{l}\frac{p}{m^{*}} \cdot 10^{-56}, \\
{\left[\mathrm{M}^{3} \cdot \mathrm{\kappa \Gamma}\right]^{-1}}\end{array}$ \\
\hline 80 & 8.20 & 54 & 0.52 & 1.25 \\
150 & 7.52 & 60 & 0.32 & 1.17 \\
200 & 6.91 & 62 & 0.21 & 1.02 \\
300 & 6.21 & 64 & 0.07 & 0.85
\end{tabular}

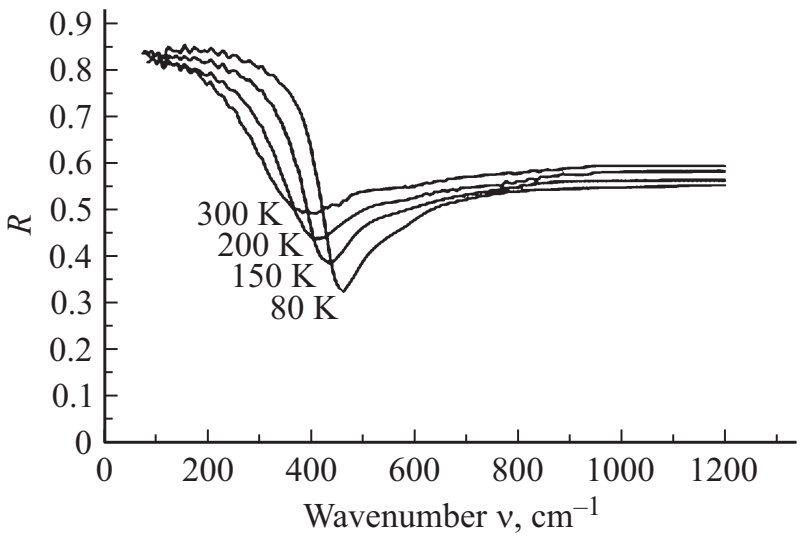

Рис. 1. Спектры коэффициента отражения $R$ монокристалла $\mathrm{Bi}_{1.5} \mathrm{Sb}_{0.5} \mathrm{Te}_{3}$ при различных температурах. Вектор напряженности электрического поля электромагнитной волны $\mathbf{E}$ перпендикулярен тригональной оси кристалла $C_{3}$.

Температурные зависимости ряда физических величин, характеризующих состояние электронной системы полупроводниковых кристаллов твердых растворов $\mathrm{Bi}_{2} \mathrm{Te}_{3}-\mathrm{Sb}_{2} \mathrm{Te}_{3}$, также указывают на уменьшение отношения концентрации к эффективной массе свободных носителей заряда $p / m^{*}$ с ростом температуры. Например, электропроводность в кристалле $\mathrm{Bi}_{1.5} \mathrm{Sb}_{0.5} \mathrm{Te}_{3}$, снижается в диапазоне от 80 до $300 \mathrm{~K}$ в 7.4 раза [2], что, с учетом температурного изменения времени релаксации за счет усиления рассеяния носителей заряда на акустических колебаниях кристаллической решетки в 5.1 раза, свидетельствует об уменьшении отношения $p / m^{*}$ в 1.45 раза [3].

В случае простого строения валентной зоны и зоны проводимости, когда в каждой из них имеется только по одному экстремуму, уменьшение отношения $p / m^{*} \mathrm{c}$ ростом температуры обычно объясняется увеличением эффективной массы носителей заряда за счет теплового расширения, а также возможной непараболичностью энергетического спектра. В случае сложного строения зоны, когда имеется несколько экстремумов, в которых находятся носители с различной эффективной массой, уменьшение отношения $p / m^{*}$ с ростом температуры может быть объяснено также и перераспределением носителей между этими подзонами, в результате чего концентрация свободных носителей заряда практически не изменяется, однако увеличивается их усредненная эффективная масса. Такое объяснение используется для интерпретации наблюдающегося в кристаллах $\mathrm{Bi}_{2} \mathrm{Te}_{3}-\mathrm{Sb}_{2} \mathrm{Te}_{3} n$ - и $p$-типа аномального температурного увеличения коэффициента Холла [1]. Например, на рис. 2 такая зависимость $R_{\mathrm{H}}$ наблюдается в $n$-типе $\mathrm{Bi}_{2} \mathrm{Te}_{3}$ в диапазоне температур от 100 до $290 \mathrm{~K}$.

Для кристаллов $p$-типа предполагается, что по мере роста температуры и заполнения дырками тяжелой подзоны ее относительная роль увеличивается, вследствие чего максимальное значение коэффициента Холла будет 


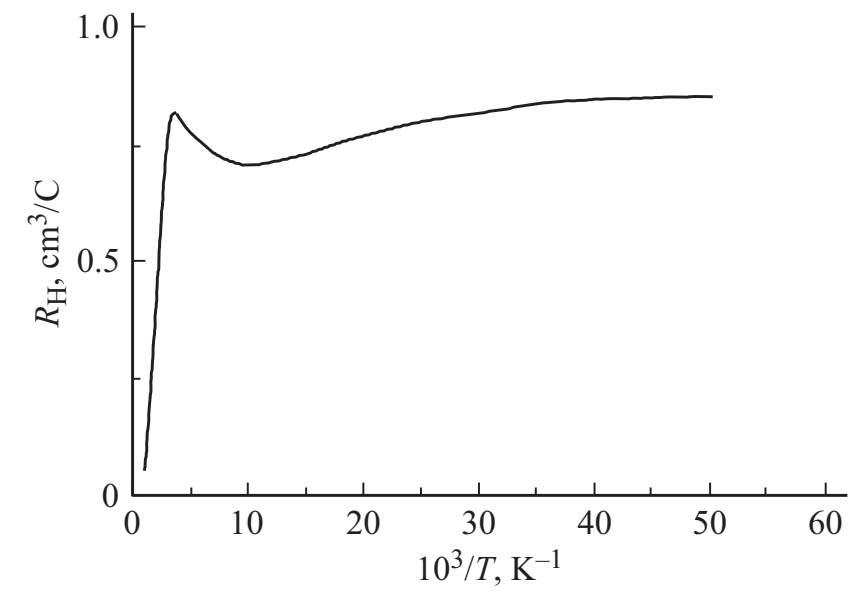

Рис. 2. Температурная зависимость коэффициента Холла $R_{\mathrm{H}}$ кристалла $\mathrm{Bi}_{2} \mathrm{Te}_{3} n$-типа, с холловской концентрацией свободных носителей заряда $1.2 \cdot 10^{19} \mathrm{~cm}^{-3}$, определенной при $T=20 \mathrm{~K}[1]$.

соответствовать температуре, при которой проводимости в подзонах легких и тяжелых дырок совпадают по величине. При дальнейшем повышении температуры коэффициент Холла будет определяться в основном подзоной тяжелых дырок, и так как концентрация в ней с ростом температуры увеличивается, то $R_{\mathrm{H}}$ уменьшается. Другие предлагаемые объяснения аномального температурного поведения коэффициента Холла, связанные, например, с влиянием степени вырождения носителей заряда или величины анизотропии времени релаксации, при переходе от примесного механизма рассеяния к фононному, вряд ли могут дать исчерпывающее объяснение аномального поведения коэффициента Холла, поскольку в кристаллах $\mathrm{Sb}_{2} \mathrm{Te}_{3}$ четырехкратное увеличение $R_{\mathrm{H}}$ наблюдается в диапазоне от 100 до $600 \mathrm{~K}$ [1]. Определенные трудности существуют и у подхода, связывающего аномальное поведение $R_{\mathrm{H}}$ с влиянием перераспределения носителей между подзонами. Действительно, в этом случае не находит объяснения отсутствие заметного роста коэффициента Холла с температурой в самых чистых образцах $n-\mathrm{Bi}_{2} \mathrm{Te}_{3}[1]$.

Так как температурные зависимости любой физической величины, связанной с концентрацией и эффективной массой свободных носителей заряда должны нести на себе отпечаток процесса, ответственного за аномальное увеличение коэффициента Холла и уменьшение отношения $p / m^{*}$ с ростом температуры, они могут быть использованы для анализа причин этих явлений. В частности, в настоящее время в ходе исследования физических свойств чистых и легированных примесями донорного и акцепторного типа кристаллов твердых растворов $\mathrm{Bi}_{2} \mathrm{Te}_{3}-\mathrm{Sb}_{2} \mathrm{Te}_{3}$, выполненных в диапазоне температур от 4.2 до $300 \mathrm{~K}$, получены новые экспериментальные данные [4]. В связи с этим оказывается возможным описание закономерностей, например, в температурных зависимостях электропроводности $\sigma$.
Одна из таких закономерностей заключается в том, что как только электропроводность исследованных в работе [4] кристаллов при температуре $15 \mathrm{~K}$ становится $<5 \cdot 10^{5} \mathrm{CM} / \mathrm{M}$, происходит изменение характера ее температурной зависимости. В кристаллах с электропроводностью $<5 \cdot 10^{5} \mathrm{CM} / \mathrm{M}$ температурная зависимость электропроводности в диапазоне от $15 \mathrm{~K}$ до температур начала собственной проводимости имеет монотонно убывающий вид - первый тип $\sigma(T)$. В кристаллах с электропроводностью $>5 \cdot 10^{5} \mathrm{CM} / \mathrm{M}$, в том же интервале температур обнаруживается более значительное и немонотонное уменьшение электропроводности - второй тип $\sigma(T)$. Как следует из экспериментальных данных, представленных в работах [2,4], переход от первого типа ко второму может быть осуществлен посредством внесения легирующей примеси или изменения соотношения компонент $\mathrm{Bi}_{2} \mathrm{Te}_{3}$ и $\mathrm{Sb}_{2} \mathrm{Te}_{3}$ в составе твердого раствора $\mathrm{Bi}_{2} \mathrm{Te}_{3}-\mathrm{Sb}_{2} \mathrm{Te}_{3}$. Особенностью температурных зависимостей $\sigma(T)$ второго типа является увеличение электропроводности в диапазоне от 4.2 до $15 \mathrm{~K}$, наблюдающееся, например, в кристаллах $\mathrm{Bi}_{0.5} \mathrm{Sb}_{1.5} \mathrm{Te}_{3}$ и $\mathrm{Sb}_{2} \mathrm{Te}_{3}$, отличающихся повышенной величиной $\sigma$, которая при $4.2 \mathrm{~K}$ превышает значение $1.5 \cdot 10^{6} \mathrm{CM} / \mathrm{M}$. Причем в кристалле $\mathrm{Sb}_{2} \mathrm{Te}_{3}$ электропроводность в интервале от 4.2 до $15 \mathrm{~K}$ увеличивается на $16 \%$, а в кристалле $\mathrm{Bi}_{0.5} \mathrm{Sb}_{1.5} \mathrm{Te}_{3}$ - на $35^{\circ}$. Рост электропроводности в кристаллах $\mathrm{Sb}_{2} \mathrm{Te}_{3}$ и $\mathrm{Bi}_{0.5} \mathrm{Sb}_{1.5} \mathrm{Te}_{3}$ в интервале от 4.2 до $15 \mathrm{~K}$ сложно объяснить увеличением времени релаксации или перераспределением носителей между подзонами. Возможно, что он связан с увеличением концентрации свободных носителей заряда. Предположим, что рост концентрации носителей в интервале от 4.2 до $15 \mathrm{~K}$ обусловлен малым потенциалом ионизации примесных атомов. Оценки, выполненные для кристалла $\mathrm{Sb}_{2} \mathrm{Te}_{3}$, изготовленного из материалов, содержащих неконтролируемую примесь в количестве $1 \cdot 10^{-4}$ ат\%, показывают, что при плотности 6.57 г/ $\mathrm{cm}^{-3}$ на $3 \cdot 10^{22}$ атомов, входящих в состав молекулы $\mathrm{Sb}_{2} \mathrm{Te}_{3}$ и находящихся в $1 \mathrm{~cm}^{-3}$, может быть $3 \cdot 10^{18}$ атомов примеси. Будем считать, что это предельное значение концентрации примесных носителей заряда, поскольку неконтролируемая примесь может состоять из компонентов донорного и акцепторного типа, компенсирующих друг друга. В этом случае увеличение концентрации, а следовательно, и величины электропроводности может быть примерно на порядок меньше наблюдаемой концентрации носителей заряда в кристалле $\mathrm{Sb}_{2} \mathrm{Te}_{3}$, которая при температуре $4.2 \mathrm{~K}$ равна $2.9 \cdot 10^{19} \mathrm{~cm}^{-3}$. Это позволяет утверждать о незначительном влиянии ионизации примесных атомов на увеличение электропроводности на 35\%, наблюдающейся в кристалле $\mathrm{Bi}_{0.5} \mathrm{Sb}_{1.5} \mathrm{Te}_{3}$ в диапазоне от 4.2 до $15 \mathrm{~K}$. Тем более что в кристалле $\mathrm{BiSbTe}_{3}$, выращенном из тех же исходных материалов, что и кристалл $\mathrm{Bi}_{0.5} \mathrm{Sb}_{1.5} \mathrm{Te}_{3}$, электропроводность в интервале от 4.2 до $15 \mathrm{~K}$ не увеличивается, а незначительно уменьшается. 
В связи с этим можно сделать вывод о том, что в кристаллах $\mathrm{Bi}_{2} \mathrm{Te}_{3}-\mathrm{Sb}_{2} \mathrm{Te}_{3}$, в которых величина электропроводности превышает $1.5 \cdot 10^{6} \mathrm{CM} / \mathrm{M}$ при $4.2 \mathrm{~K}$, интенсифицируется некоторый процесс, ведущий к росту концентрации свободных носителей заряда и, соответственно, электропроводности в диапазоне от 4.2 до $15 \mathrm{~K}$. Последующее уменьшение величины электропроводности с ростом температуры от $15 \mathrm{~K}$ и до начала доминирования собственной проводимости, сопровождающееся аномальным увеличением коэффициента Холла, может быть частично обусловлено снижением интенсивности указанного процесса.

Чтобы приблизиться к пониманию природы этого процесса, будем исходить из того, что существуют несколько механизмов формирования подсистемы свободных носителей заряда. Один из них связан с разрывом ковалентных связей тепловыми колебаниями ионного остова, а другой - с перекрытием энергетических зон сближающихся атомов, образующих конденсированное состояние.

Кроме этого, как было показано еще Моттом [5], в определенных условиях возможны фазовые переходы типа металл-диэлектрик, сопровождающиеся существенным изменением электропроводности материала и характера температурной зависимости электропроводности. Существует несколько теоретических подходов к описанию природы переходов Мотта. Один из них связан с предположением о том, что изменение температуры или давления может привести к изменению количества связанных состояний электронов с ионным остовом, которое происходит благодаря изменению интенсивности экранирования кулоновского взаимодействия слабо связанных электронов с ионным остовом, другими электронами проводимости [6], образующими плазму свободных носителей заряда.

Другой подход к описанию природы переходов Мотта основан на предположении об изменении величины прямой энергетической щели под воздействием внешних факторов [6]. Это согласуется с доводами, приведенными в работе [7], в которой утверждается, что атомная энергия ионизации может уменьшаться за счет обратного воздействия поляризации плотно упакованных атомов, в общем пространстве которых существуют плазменные колебания носителей заряда, смещающихся как единое целое относительно ионного остова, и тем самым создающих дипольный момент. Такие колебания принято рассматривать как плазмон-фонон-поляритоны. Из рассмотрения динамической поляризуемости конденсированного вещества следует, что атомная энергия ионизации $E_{0}=\hbar \omega_{0}$ уменьшается за счет обратного воздействия поляризации до величины $E_{g}=\hbar \omega_{g}$. Как показано в работе [7], величину частоты $\omega_{g}$ можно оценить в соответствии с выражением

$$
\omega_{g}^{2}=\omega_{0}^{2}-\frac{\omega_{p v}^{2}}{3}
$$

в котором $\omega_{0}$ - частота, соответствующая атомной энергии ионизации, а $\omega_{p v}$ - плазменная частота ва- лентных электронов. Таким образом, просматривается принципиальная возможность зависимости величины энергетической щели от частоты плазменных колебаний. В этом случае температурная зависимость величины дипольного момента, обусловленного наличием плазмонфонон-поляритонов, может вносить вклад в изменение энергетических параметров зонной структуры полупроводникового материала и способствовать немонотонному температурному поведению параметров, характеризующих состояние электронной системы.

\section{4. Заключение}

Как следует из представленных экспериментальных данных, в кристаллах полупроводников $\mathrm{Bi}_{2} \mathrm{Te}_{3}-\mathrm{Sb}_{2} \mathrm{Te}_{3}$, как и в ряде других узкозонных материалов, отличающихся изначально высокой концентрацией свободных носителей заряда, наблюдаются плазменные колебания, резонансные частоты которых близки к $\omega_{g}$, что предопределяет возможность существования плазмон-фононполяритонов и интенсификации процесса обратного воздействия поляризации, именно при низких температурах. С увеличением температуры влияние этого процесса будет становиться менее эффективным, вследствие усиления хаотизации, что приведет к ослаблению экранирования кулоновского взаимодействия плазмонфонон-поляритонами. Как следствие этого, может интенсифицироваться процесс возникновения связанных состояний электронов с ионным остовом, появление которых наряду с другими возможными процессами, описанными выше, будет являться причиной уменьшения величины электропроводности, а также аномального увеличения коэффициента Холла с ростом температуры.

\section{Список литературы}

[1] Б.М. Гольцман, В.А. Кудинов, И.А. Смирнов. Полупроводниковые термоэлектрические материалы на основе $\mathrm{Bi}_{2} \mathrm{Te}_{3}$ (М., Наука, 1972) гл. 5, с. 114.

[2] Л.Д. Иванова, Ю.В. Гранаткина. Неорг. матер., 36, 810 (2000).

[3] Н.П. Степанов, Л.Э. Степанова, А.С. Лозовская. Изв. вузов. Физика, 61, 55 (2018).

[4] А.А. Кудряшов. Влияние легирования на термоэлектрические свойства и эффект Шубникова-де Гааза твердых растворов теллуридов и селенидов висмута и сурьмы: дис. канд. физ.-мат. наук: $01.04 .09-$ Физика низких температур (М., МГУ, 2016) $101 \mathrm{c}$.

[5] N.F. Mott. Can. J. Phis., 34, 1356 (1956).

[6] Ч. Киттель. Введение в физику твердого тела (М., Наука, $1978)$ гл. 6 , с. $227,740$.

[7] П. Гроссе. Свободные электроны в твердых телах (М., Мир, 1982) гл. 1, с. 33.

Редактор Г.А. Оганесян 
The nature of the anomalous temperature

dependence of the Hall coefficient

observed in semiconductor crystals

of $\mathrm{Bi}_{2} \mathrm{Te}_{3}-\mathrm{Sb}_{2} \mathrm{Te}_{3}$ solid solutions

N.P. Stepanov

Transbaikal State University,

672039 Chita, Russia

Abstract Based on the analysis of experimental results, it is shown that in crystals of $\mathrm{Bi}_{2} \mathrm{Te}_{3}-\mathrm{Sb}_{2} \mathrm{Te}_{3}$, in which the electrical conductivity exceeds $1.5 \cdot 10^{6} \mathrm{~S} / \mathrm{m}$ at $4.2 \mathrm{~K}$, a certain process is responsible for an increase of electrical conductivity in the temperature range between 4.2 and $15 \mathrm{~K}$. With further increase of the temperature up to the intrinsic conductivity dominance, electrical conductivity decreases and an abnormal increase of the Hall coefficient is observed, which indicates the diminishing role of the aforementioned process. In this letter we suggest that in conditions of plasma energy and band gap width convergence, this process comes as a result of the inverse polarization effect provided by surface plasmon polaritons. 